\title{
Potentiometric back titration as a robust and simple method for specific surface area estimation of lignocellulosic fibers
}

\author{
Ferran Serra-Parareda - Roberto Aguado - Quim Tarrés · Pere Mutjé • \\ Marc Delgado-Aguilar
}

Received: 18 May 2021 / Accepted: 1 October 2021 / Published online: 13 October 2021

(C) The Author(s) 2021

\begin{abstract}
The specific surface area (SSA) of cellulosic or lignocellulosic fibers is seldom reported in the recent literature on papermaking, despite its close relation with the degree of refining and other key pulp properties. Amidst outdated assays (Pulmac permeability test) and methods that, while accurate, are of doubtful usefulness for papermaking purposes $\left(\mathrm{N}_{2}\right.$ adsorption-desorption), we suggest a methodology based on the cationic demand. A commonly used cationic polyelectrolyte, poly(diallyldimethylammonium chloride) (PDADMAC), became adsorbed onto thermomechanical pulp samples. Then, a potentiometric back titration with an anionic polyelectrolyte measured the cationic demand, expressed as microequivalents of PDADMAC per gram of pulp. Multiplying this value by the surface area of a microequivalent of polymer, considering rod-like conformation in the case of minimum ionic strength, yielded the SSA of the lignocellulosic pulp. Our system assumes that the quaternary ammonium groups were anchored through electrostatic and ion-dipole
\end{abstract}

F. Serra-Parareda · Q. Tarrés · P. Mutjé .

M. Delgado-Aguilar ( $\square)$

LEPAMAP-PRODIS Research Group, University of Girona, C/ Maria Aurèlia Capmany, 61, 17003 Girona, Spain

e-mail: m.delgado@udg.edu

R. Aguado

Department of Chemistry, CQC, University of Coimbra, Rua Larga, 3004-535 Coimbra, Portugal interactions. Measuring the carboxyl content allowed for discriminating between both kinds of forces. Finally, the model could be validated by plotting the estimated SSA values against the Schopper-Riegler degree, attaining high correlation coefficients $\left(\mathrm{R}^{2} \sim 0.98\right)$. Owing to the high molecular weight of the polyelectrolyte of choice $(107 \mathrm{kDa})$, and more particularly in the case of fine-free pulps, SSA values estimated from the cationic demand were consistently lower than those from dye (Congo red) sorption. Instead of being a drawback, the limited diffusion of PDADMAC through fibers can enable papermakers to attain a more helpful quantification of the available surfaces in operations with low residence times.

Keywords Cationic demand $\cdot$ Lignocellulosic fibers · Papermaking · Polydadmac - Specific surface area

\section{Introduction}

The wall of natural plant fibers, consisting essentially of $\alpha$-cellulose, hemicelluloses and lignin, is smooth and hardly porous in the absence of water, thus displaying a low surface area (Topgaard and Söderman 2002). This can change drastically along the papermaking process, particularly during pulping and refining (Aguado et al. 2016; Przybysz et al. 2020). 
Indeed, the surface area of fibers, both inner and outer, is of utmost importance in papermaking, among other reasons because it strongly influences bulk density, air permeability, and water absorbency (Koponen et al. 2017; Mao et al. 2017; Azevedo et al. 2020). Furthermore, as paper strength is largely due to hydrogen bonding and other non-covalent interactions in pulps, the availability of a large area for bonding is generally translated into good mechanical properties (Motamedian et al. 2019). Not less importantly, this available area is reduced by hornification, a side effect in the structure of fibers as a consequence of the recycling process, mainly the subsequent drying and wetting stages (Delgado-Aguilar et al. 2015; Moser et al. 2018).

In the case of chemical pulping, pores are created by dissolving the hemicellulose and lignin fractions of fibers (Topgaard and Söderman 2002). And while these fractions remain after high-yield pulping, swelling still takes place through the hydration of fibers, especially in thermomechanical pulping (TMP) (Moral et al. 2017; Serra-Parareda et al. 2021). Nonetheless, the presence of lignin interferes with intra- and interfiber bonding, which makes a refining stage more necessary. During refining, fibers undergo shortening (or cutting), partial removal of the wall, redistribution of hemicelluloses, and fibrillation (Gharehkhani et al. 2015; Espinosa et al. 2018; Przybysz et al. 2020). External and internal fibrillation increase the outer and inner surface areas, respectively. The former is due to fibrils protruding from the surface of fibers, which becomes rougher (Moral et al. 2016), whereas the latter is evidenced by swelling (Lecourt et al. 2010). When it comes to TMP fibers, their negative charges (owing mostly to carboxyl groups) become more openly exposed (Zhao et al. 2016).

Despite the usefulness of measuring the specific surface area (SSA), this property is seldom reported in the context of papermaking, at least as of today. Earlier, the Pulmac permeability test was more commonly used for this purpose (Robertson and Mason 1949; Rouger and Mutjé 1984; Carrasco et al. 1996). It used to be a troublesome measurement, lacking reproducibility and reliability (Ramarao 1999). Another popular liquid-solid method implies the adsorption of dyes, such as methylene blue and Congo Red, onto the available surfaces of fibers, and then recording electronic absorption spectra (Kaewprasit et al. 1998).

It is in regards of cellulose-based sorbent materials and cellulose nanofibers that the SSA is often reported in recent works. This calculation of the SSA has preferentially used $\mathrm{N}_{2}$ adsorption isotherms, and more specifically the BET model (Nemr et al. 2017; Hina et al. 2018; Darpentigny et al. 2020). While there is no doubt about the suitability of the BET method for this measurement, it requires specific equipment, often named "surface area analyzer". Furthermore, a monolayer of adsorbed $\mathrm{N}_{2}$ is formed all across the porous structure of the material, including those pores whose size is as small as $2 \mathrm{~nm}$. Hence, the information gathered is extremely useful for nanocellulose-based gels (Tarrés et al. 2016; Darpentigny et al. 2020), but it would be not so relevant for the paper manufacturing process, which takes place in aqueous media and involving low residence times.

In this work, we evaluate a potentiometric titration with a cationic polyelectrolyte, poly(diallyldimethylammonium chloride) (PDADMAC), as a practical and reliable way to measure the SSA of TMP fibers. The surface area of fibers from their cationic demand has been previously estimated in previous works (Tarrés et al. 2018; Filipova et al. 2020), but no work justifying the method on the basis of an in-depth study and strong correlations has been published yet. It is worth highlighting a valuable contribution from Zhang et al. (2016), who showed that the cationic demand measured by researchers actually depends on the molecular weight (MW) of PDADMAC. This inspires us to infer that PDADMAC of medium or high MW can be easily adsorbed along the outer surfaces of lignocellulosic fibers and can diffuse through relatively large pores, not reaching the small pores to which $\mathrm{N}_{2}$ and low-MW dyes can adsorb. Instead of a drawback, the SSA value estimated from a titration with high-MW PDADMAC is hypothesized to correspond more faithfully to the actual bonding area of fibers in the fast processes of a paper mill. This estimation of the SSA could then be used to quantify the degree of refining, to monitor hornification as a result of recycling, and to predict paper wet strength. 


\section{Experimental}

Materials

PDADMAC with an average MW of $107 \mathrm{kDa}$ was kindly supplied by L.C. Paper (Besalú, Spain). The source of lignocellulosic fibers was a TMP from softwood, of industrial origin, that had undergone chlorine-free bleaching and whose freeness was $21.5^{\circ} \mathrm{SR}$. Sodium polyethylene sulfonate (PES-Na) was provided by BTG Instruments. Congo red (CR) and all other reagents were purchased from SigmaAldrich.

\section{Refining}

$30 \mathrm{~g}$ of pulp (on the basis of dry pulp weight) were dispersed in a pulp disintegrator at a consistency of $1.5 \%$, for $10 \mathrm{~min}$ at $3,000 \mathrm{rpm}$. The suspension was filtered so as to adjust its consistency to $10 \%$ (wt), and then refined in a PFI mill from Metrotec, model NPFI 02 , according to ISO 5264-2. TMP was refined to 5,000, 10,000, 20,000, and 30,000 PFI revolutions. The energy input was quantified by means of a device from Circutor, model CVM-C10.

Freeness of pulp was measured by means of a Schopper-Riegler tester, 95,587 PTI, in accordance to the ISO standard 5267-1. Canadian Standard Freeness (CSF) was interpolated from a freeness conversion table (González et al. 2012). Moreover, the water retention value (WRV) was measured gravimetrically, in accordance to the SCAN-C method 62:00. Fiber pads containing excess water were centrifuged at $3000 \mathrm{~g}$ in containers provided with a nitrocellulose membrane $(0.22 \mu \mathrm{m}$ of pore size), and by means of a Sigma Laborzentrifugen apparatus, model 6K15. After $15 \mathrm{~min}$, the filter cake was collected, weighted $\left(\mathrm{m}_{\mathrm{W}}\right)$, and oven-dried at $105{ }^{\circ} \mathrm{C}$ until constant weight $\left(m_{D}\right)$. Then, WRV equals the relative difference between those weights:

$W R V=\left(m_{W}-m_{D}\right) / m_{D}$

\section{Adsorption of Congo red}

After verifying the compliance of $\mathrm{CR}$ to the BeerLambert's law, by plotting the absorbance at a wavelength of $488 \mathrm{~nm}$ against the concentration, adsorption experiments were carried out as reported elsewhere (Inglesby and Zeronian 2002). Briefly, TMP samples were suspended in phosphate buffer $(0.1 \mathrm{M})$ at $\mathrm{pH} 6$ and mixed with varying amounts of $\mathrm{CR}$, from 5 to $15 \%$ (wt.), then stored at $60{ }^{\circ} \mathrm{C}$ for $24 \mathrm{~h}$. We added $\mathrm{NaCl}(0.004 \%$, wt. $)$ and centrifuged the samples for $20 \mathrm{~min}$. Then, UV-visible absorption spectra were recorded from the free liquid and the concentration of CR in each case was computed. The maximum adsorption capacity $\left(q_{\max }\right)$ was calculated by fitting to a linearized Langmuir isotherm:

$\frac{c_{e q}}{q}=\frac{1}{K_{a d} q_{\max }}+\frac{c_{e q}}{q_{\max }}$

where $c_{e q}$ is the concentration of $\mathrm{CR}$ at equilibrium $(\mathrm{mg} / \mathrm{L}), q$ is the adsorbed mass of CR $(\mathrm{mg} / \mathrm{g})$, and $K_{a d}$ is the equilibrium constant. Then, the SSA can be estimated from:

$S S A_{C R}=\frac{q_{\max } \times N_{A} \times S A_{C R}}{10^{21} M w_{C R}}$

where $N_{A}$ is the Avogadro number, $S A_{C R}$ is the surface area of a single molecule of adsorbate $\left(1.73 \mathrm{~nm}^{2}\right)$, and $M w_{C R}$ is the molecular weight of CR $(696.7 \mathrm{~g} / \mathrm{mol})$.

Carboxyl content and cationic demand

Carboxyl groups were quantified by means of a conductimetric titration. A sample of TMP was suspended in $15 \mathrm{~mL}$ of $\mathrm{HCl} 0.01 \mathrm{M}$. This suspension was titrated with an aqueous $\mathrm{NaOH}$ solution, continuously recording the conductivity values. Then, we plotted a titration curve, which encompassed two inflection points, one being due to the strong acid $(\mathrm{HCl})$ and the other one being due to weak acids (carboxyl groups). Then, the carboxyl content ( $C C$, in mmol per gram of dry sample) is calculated from:

$C C=\left(V_{2}-V_{1}\right) \times c / m$

where $V_{1}$ and $V_{2}$ are the volumes of $\mathrm{NaOH}(\mathrm{mL})$ that corresponded to the first inflection point of the curve and the equivalence point, respectively; $c$ is the concentration of $\mathrm{NaOH}(10 \mathrm{mM})$, and $m$ is the mass of pulp (on a dry basis) in the suspension.

The cationic demand was determined potentiometrically, performing a back titration with a particle charge detector Mütek PCD-04 (BTG Instruments). $0.1 \mathrm{~g}$ of dry TMP were mixed with a known excess of 
PDADMAC, typically $10 \mathrm{~mL}\left(V_{P D A D M A C}\right)$, and in deionized water medium. Other than the cationic polyelectrolyte and its counter-ion $\left(\mathrm{Cl}^{-}\right)$, the presence of ionic species can be neglected, given that the pulp had been thoroughly washed with deionized water. The suspension was centrifuged for $30 \mathrm{~min}$ at $10,000 \mathrm{rpm}$. Then, the supernatant was titrated with PES-Na until the isoelectric point $(0 \mathrm{mV})$. The cationic demand $(C D)$ was then determined as:

$$
C D=\left(c_{P D A D M A C} \times V_{P D A D M A C}\right)-\left(c_{P E S-N a} \times V_{P E S-N a}\right)
$$

where $c_{P D A D M A C} c_{P o l y D A D M A C}$ is the normal concentration of PDADMAC (typically $0.001 \mathrm{~N}$ ), $c_{P E S-N a}$ $c_{P E S-N a}$ is the normal concentration of titrating agent (typically $0.001 \mathrm{~N}$ ), and $V_{P E S-N a} V_{P E S-N a}$ is the titration volume.

The hypothesis that these cationic demand measurements can be correlated to SSA, along with other properties that are also affected by refining, was evaluated by calculating the Pearson correlation coefficient.

Furthermore, the influence of the ionic strength on PDADMAC adsorption was assessed by dissolving different amounts of $\mathrm{NaCl}$ in the medium, before interacting with the pulp. Likewise, small additions of diluted $\mathrm{NaOH}$ or diluted $\mathrm{HCl}$ allowed us to study the effect of $\mathrm{pH}$.

\section{Separation of fines}

In this work, fines are understood as those particles in a cellulosic or lignocellulosic pulp whose length lies below $75 \mu \mathrm{m}$. The consistency of a TMP suspension was adjusted to $1 \%$ and it was filtered through a 200-mesh screen. The fraction that passed through the screen was regarded as the fines content, which was determined gravimetrically, on the basis of dry pulp weight. Fibers, on the other hand, remained onto the mesh screen.

Then, the aforementioned procedures to estimate the surface area from PDADMAC adsorption, including measurements of the carboxyl content and the cationic demand, were performed separately for fibers and for fines. For comparison purposes, the Congo red sorption method was also carried out.

\section{Modeling}

Assumptions on polyelectrolyte-fiber interactions

Significant interactions between PDADMAC and lignocellulosic fibers, at least in an aqueous medium, can plausibly be reduced to intermolecular forces between hydrophilic functional groups. The carboxyl groups of lignin and certain hemicelluloses (comprising hexuronic acid, penturonic acid and hexenuronic acid units) become strongly attracted to the quaternary ammonium groups of PDADMAC. Besides these electrostatic interactions between charged groups, hydroxyl groups can be polarized in such a way that their hydrogen atom acquires partial positive charge and their oxygen atom acquires partial negative charge (Barrera and Jorge 2020). Therefore, quaternary ammonium groups are also attracted to the hydroxyl groups of cellulose, hemicelluloses and lignin by iondipole interactions. This is shown in Fig. 1.

Meanwhile, hydrophobic interactions occur between cellulose chains, between them and lignin, and between them and hemicellulose (Alves et al. 2015; Aguado et al. 2019), but such interactions can be neglected for PDADMAC, considering its highly hydrophilic behavior. While, especially due to the methylene $\left(-\mathrm{CH}_{2}-\right)$ groups, PDADMAC is capable of establishing hydrophobic interactions, they are expected to be, comparatively speaking and under the conditions of these assays, much less intense than Coulomb forces. Likewise, $-\mathrm{OH}$ and $-\mathrm{COOH}$ groups take part in hydrogen bonds between them and with water, but quaternary ammonium groups are not capable of hydrogen bonding, neither as acceptors nor as donors. Finally, dipole-dipole attraction forces

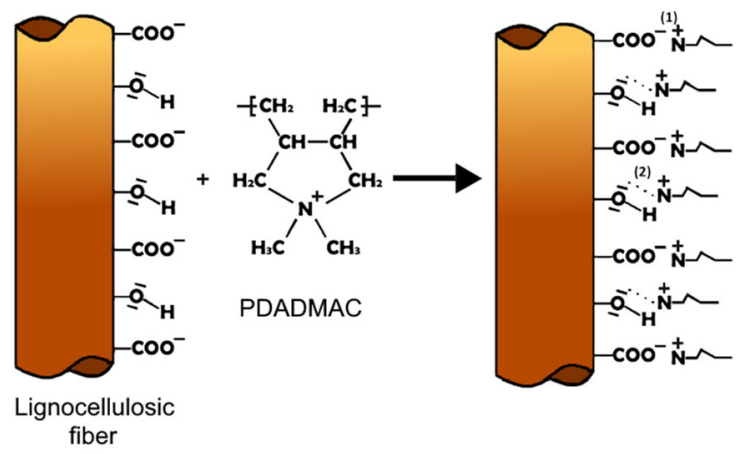

Fig. 1 Electrostatic (1) and ion-dipole interactions (2) of PDADMAC with lignocellulosic fibers, such as those from TMP 
cannot be ruled out, but they play a minor role in comparison to electrostatic and ion-dipole interactions.

The cationic demand, as determined by the aforementioned potentiometric titration, is expressed as equivalents of polyelectrolyte per gram of pulp. Considering, then, that each PDADMAC monomer has only one anchoring group to be immobilized onto fibers, $1 \mu$ eq of polyelectrolyte corresponds to $1 \mu \mathrm{mol}$ of cationic monomeric units that remained in the pulp sample, retained by carbohydrates and lignin, after centrifugation. The high molecular weight of PDADMAC $(>100 \mathrm{kPa})$ implies kinetic and equilibrium limitations to its diffusion through fibers (Zhang et al. 2016), and thus adsorption is expected to take place primarily at surfaces and relatively large pores. Far from being a drawback, this kind of interaction with fibers may resemble that of the polycations used in papermaking processes.

Furthermore, as assumed for CR, PDADMAC is hypothesized to form a monolayer over the available surfaces of the fiber. The monolayer assumption implies neglecting chain entanglement and attraction between like-charged macromolecules. In any case, deviations from these assumptions would affect the interpretation of results (e.g., overestimating the ease of diffusion of PDADMAC through the fiber), but not the goodness and usefulness of correlations with SSA, the degree of refining, and other properties of interest.

\section{Understanding the structure of PDADMAC}

The semi-structural formula of a PDADMAC monomer is displayed in Fig. 2. All carbon atoms present an $\mathrm{sp}^{3}$ hybridization, meaning that $\mathrm{C}-\mathrm{C}-\mathrm{C}, \mathrm{C}-\mathrm{C}-\mathrm{H}$ and $\mathrm{H}-\mathrm{C}-\mathrm{H}$ angles are ideally $109.5^{\circ}$. Likewise, the orbitals of nitrogen are $\mathrm{sp}^{3}$-hybridized and form a sigma bond with each surrounding carbon, so the same can be said of $\mathrm{C}-\mathrm{N}-\mathrm{C}$. Asymmetry and ring strain (due to the cycle) necessarily imply a deviation from that angle, but we can neglect such variation on the basis of two reasons. First, each of the angles of an equiangular pentagon is $108^{\circ}$, very close to $109.5^{\circ}$. Second, there are no lone pairs of electrons that could impart a great deviation.

The average internuclear distances used in this model for $\mathrm{C}-\mathrm{C}, \mathrm{C}-\mathrm{N}$ and $\mathrm{C}-\mathrm{H}$ are $1.54,1.43$ and $1.10 \AA$, respectively (Carey and Sundberg 2007). From these values and trigonometric calculations,

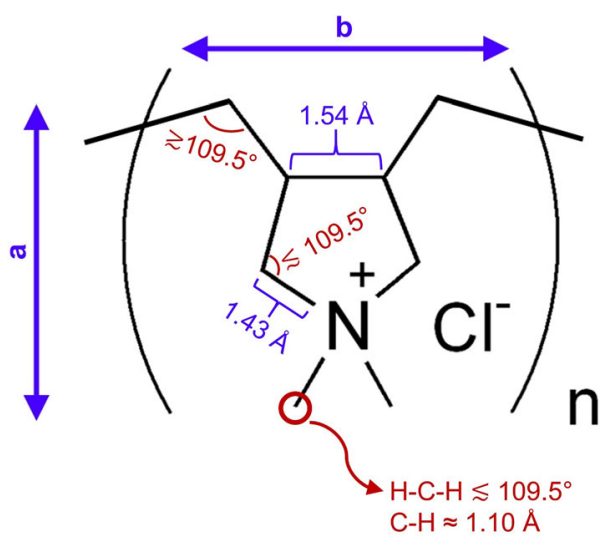

Fig. 2 Structure of the PDADMAC monomer, indicating interatomic distances and approximate bonding angles

distance "a" in Fig. 2 equals $5.43 \AA$, while distance "b" is $4.85 \AA$.

A slab geometry for PDADMAC chains, taking into account that charged polymers tend to present high stiffness, is assumed for the case of minimum ionic strength (Trizac and Shen 2016; Zhang et al. 2016; Scheepers et al. 2021). Indeed, the lack of ring strain (pentagon, $108^{\circ} \sim 109.5^{\circ}$ ) justifies the assumption that each of the monomers is planar, while the aforementioned stiffness is translated into less rotation around the $-\mathrm{CH}_{2}-\mathrm{CH}_{2}-$ axis. Thence the area of a PDADMAC chain is given by:

$A_{P D A D M A C}=a \times b \times D P$

From the molar mass of the monomer and the average molecular weight of PDADMAC, the average degree of polymerization (DP) is 662 . The average area of the planar surface of this polyelectrolyte, then, equals $171 \mathrm{~nm}^{2}$. Given that $1 \mathrm{~mol}$ of monomers is $1 \mathrm{eq}$ of PDADMAC, this implies a value of $1.55 \cdot 10^{17} \mathrm{~nm}^{2}$ $\mu \mathrm{eq}^{-1}$. Finally, the specific surface of pulps, fibers or fines can be calculated from the amount of polyelectrolyte adsorbed preferentially by ion-dipole forces:

$$
\begin{aligned}
S S A_{P D A D M A C}= & (C D-C C) \times 1.55 \\
& \cdot 10^{17} \mathrm{~nm}^{2} \mu e q^{-1} \frac{1 m^{2}}{10^{18} \mathrm{~nm}^{2}}
\end{aligned}
$$

It should be noted that the chain length value used in this estimation, $321 \mathrm{~nm}$ (corresponding to the average DP, 662), is based on the Kuhn length for a rod-like model. Even though rotations around methylene-methylene would result in a lower end-to-end length (Strobl 2007), that value would still be valid to 
define the contour length. In contrast, in a worm-like model where rotations are allowed, but only by small angles, length could be roughly estimated as $160 \mathrm{~nm}$ (Zhang et al. 2019). Owing to the deionized medium and considering that the value of the constant in Eq. 7 will not affect the goodness of the correlations (Pearson's coefficients), the rod-like model and the slab geometry will be generally assumed for length and area, respectively.

\section{Results and discussion}

Validation of the methodology in refined pulps

As stated above, the estimation of the SSA value described in this work is hypothesized to quantify the level of refining. Consequently, Table 1 presents the common pulp properties that are directly related to this stage, besides the specific surface area as calculated from dye sorption $\left(\mathrm{SSA}_{\mathrm{CR}}\right)$, of TMP samples. Comparing the latter to the rest of the measurements, Pearson's $r$ is 0.93 with the number of PFI revolutions, 0.96 with energy consumption, - 0.94 with CSF, 0.98 with the fines content, and above 0.99 with both WRV and the Schopper-Riegler degree. This degree, universally used to show to what extent a pulp has been refined on the basis of its drainage behavior, can thus be used to validate the estimation of SSA as an indicator of refining.

The results from the measurements and calculations leading to the SSA PDADMAC (i.e., the specific surface area as calculated from the cationic demand) are shown in Table 2. Moreover, once the area of available surfaces per gram of pulp is known, it is possible to express important properties in terms of area density.

High correlation coefficients $\left(\mathrm{R}^{2} \sim 0.98\right)$ are obtained when plotting the SSA, regardless of how it
Table 1 Evolution of key pulp properties and energy consumption with the number of PFI revolutions during refining

\begin{tabular}{lllllc}
\hline Number of PFI revolutions & 0 & 5000 & 10,000 & 20,000 & 30,000 \\
\hline Refining degree $\left({ }^{\circ} \mathrm{SR}\right)$ & 21.5 & 58.5 & 77.8 & 91.3 & 95.5 \\
$\mathrm{CSF}(\mathrm{mL})$ & 578 & 150 & 66 & 10 & 0.6 \\
Specific Energy Consumption, SEC $(\mathrm{kWh} / \mathrm{kg})$ & - & 2.28 & 4.45 & 8.70 & 12.80 \\
Fines content (w/w \%) & 12.9 & 27.0 & 35.5 & 48.7 & 59.3 \\
$\mathrm{WRV}(\mathrm{g} / \mathrm{g})$ & 0.29 & 0.72 & 1.11 & 1.30 & 1.48 \\
$\mathrm{SSA}_{\mathrm{CR}}\left(\mathrm{m}^{2} / \mathrm{g}\right)$ & 2.87 & 10.43 & 15.88 & 19.38 & 22.10 \\
\hline
\end{tabular}

Table 2 Estimation of the surface area per gram of pulps from the potentiometric and conductimetric titrations

\begin{tabular}{|c|c|c|c|c|c|c|}
\hline \multicolumn{2}{|l|}{ Refining degree ( $\left.{ }^{\circ} \mathrm{SR}\right)$} & 21.5 & 58.5 & 77.8 & 91.3 & 95.5 \\
\hline \multicolumn{2}{|l|}{ Carboxyl content ( $\mu$ eq $\mathrm{COO}^{-} / \mathrm{g}$ pulp) } & 45.72 & 48.46 & 44.56 & 46.87 & 44.99 \\
\hline \multicolumn{2}{|l|}{ CD ( $\mu$ eq PDADMAC/g pulp) } & 56.3 & 95.5 & 124.5 & 140.9 & 154.1 \\
\hline \multicolumn{2}{|c|}{ Cationic demand of free-fine fibers, $\mathrm{CD}_{\mathrm{F}}$ ( $\mu$ eq PDADMAC/g pulp) } & 34.4 & 53.6 & 79.1 & 78.5 & 76.3 \\
\hline \multirow[t]{2}{*}{ Amount of fixed polymer ( $\mu$ eq PDADMAC/g pulp) } & Electrostatic & 41.6 & 42.2 & 43.4 & 41.9 & 41.2 \\
\hline & Ion-dipole & 14.7 & 53.3 & 81.1 & 99.0 & 112.9 \\
\hline \multicolumn{2}{|l|}{ Carboxyl/polymer ratio ( $\mu$ eq $\mathrm{COO}^{-} /$eq PDADMAC) } & 1.10 & 1.15 & 1.03 & 1.12 & 1.09 \\
\hline \multicolumn{2}{|l|}{$\mathrm{SSA}_{\text {PDADMAC }}{ }^{1}\left(\mathrm{~m}^{2} / \mathrm{g}\right)$} & 2.27 & 8.26 & 12.58 & 15.35 & 17.50 \\
\hline \multicolumn{2}{|l|}{$\operatorname{SSA}_{\text {PDADMAC }}{ }^{2}\left(\mathrm{~m}^{2} / \mathrm{g}\right)$} & 1.64 & 7.29 & 12.39 & 14.57 & 16.91 \\
\hline \multicolumn{2}{|l|}{ Carboxyl density ( $\mu$ eq $\mathrm{COO}^{-} / \mathrm{m}^{2}$ pulp) } & 15.93 & 4.65 & 2.81 & 2.42 & 2.04 \\
\hline \multicolumn{2}{|l|}{ Fixed polymer density ( $\mu$ eq PDADMAC $/ \mathrm{m}^{2}$ pulp) } & 19.62 & 9.16 & 7.84 & 7.27 & 6.97 \\
\hline
\end{tabular}

${ }^{1}$ Estimated from the total sorption of PDADMAC

${ }^{2}$ Estimated from the difference between $\mathrm{CD}$ and $\mathrm{CC}$, and considering monolayer adsorption of PDADMAC 


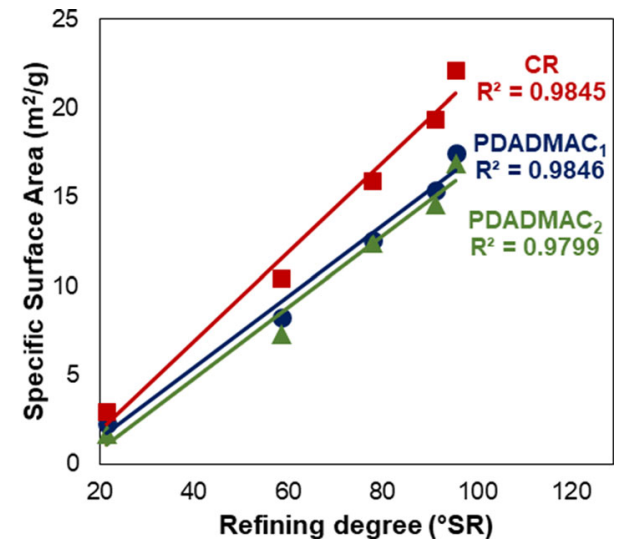

Fig. 3 Correlation between the Schopper-Riegler degree and the SSA of pulps, calculated by different methods

was estimated, against the Schopper-Riegler degree, as can be seen from Fig. 3. The estimation from CR sorption $\left(\mathrm{SSA}_{\mathrm{CR}}\right)$ yields consistently higher values, given the ease of diffusion of this low-MW compound through fibers, than the estimations from the cationic demand, both with and without subtracting the carboxyl content (SSAPDADMAC2 and SSAPDADMAC1, respectively).

Like PDADMAC, $\mathrm{CR}$ attaches to $-\mathrm{OH}$ groups through ion-dipole interactions, but in the opposite way. The partial positive charge of hydrogen atoms in those groups attracts the sulfonate groups of CR. Furthermore, in the case of lignocellulosic fibers, such as those from TMP, their ionized carboxyl groups are attracted to the amino groups of CR. The main difference, then, lies in the molecular weight. In Fickian or pseudo-Fickian diffusion, as it is wellknown, the diffusion coefficient is inversely proportional to the hydrodynamic radius of the solute, and thus to a fractional power of the molecular weight (Friedman and Mills 1986).

\section{Differences between fibers and fines}

As expected, certain effects of refining on fibers, mainly cutting or shortening, are accompanied by an increasing generation of fines with increasing number of PFI revolutions (Table 1). Discriminating between fibers and fines is precisely one of the advantages of estimating the SSA by means of compounds whose diffusion through lignocellulosics is limited. Figure 4 shows a great disparity between the SSA of the finefree fraction (Fig. 4a) and the SSA of fines (Fig. 4b).
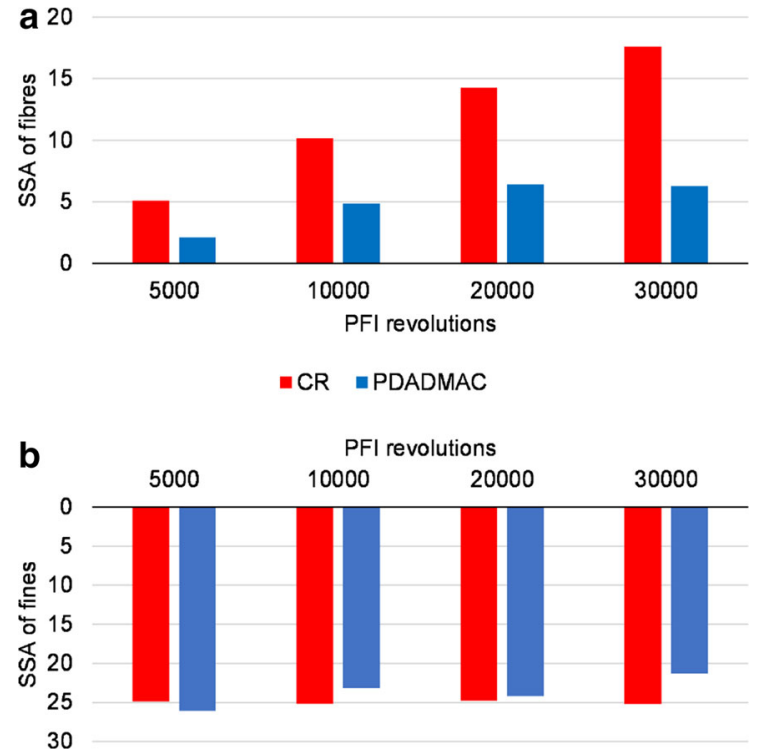

Fig. 4 Difference in SSA between fibers and fines, as a function of the number of PFI revolutions

Interestingly enough, while in fibers, the estimation from CR adsorption at least doubles that from PDADMAC (including CC subtraction), both methods yield a similar result when it comes to fines.

Figure 5 schematizes how CR molecules or PDADMAC macromolecules diffuse through fibers (Fig. 5a) and fines (Fig. 5b). In the former's case, in which diffusion can be assumed to be exclusively radial, the higher the molecular weight of the adsorbate is, the more hindered it is to reach inner surfaces, regardless of the adsorption constants. It should be noted that the presence of hemicellulose and lignin in TMP holds cellulose microfibrils together, contributing to this hindrance (Tarrés et al. 2020). In the case of fines, diffusion happens to a significant extent in more directions, and the lower hydrodynamical radius of particles reduces the influence of the molecular weight of the solute.

All things considered, the SSA of fibers estimated from a potentiometric titration with PDADMAC will be consistently lower than that the SSA estimated from dye sorption and, obviously, from the BET determination $\left(\mathrm{N}_{2}\right.$ adsorption-desorption). However, to our judgment, in the most typical wet operations of a paper mill, from refining to filtration or to coating, the estimation using PDADMAC is, instead of an underestimation, a reliable insight into the actually available surfaces. One of the reasons is that the pulp stock is 


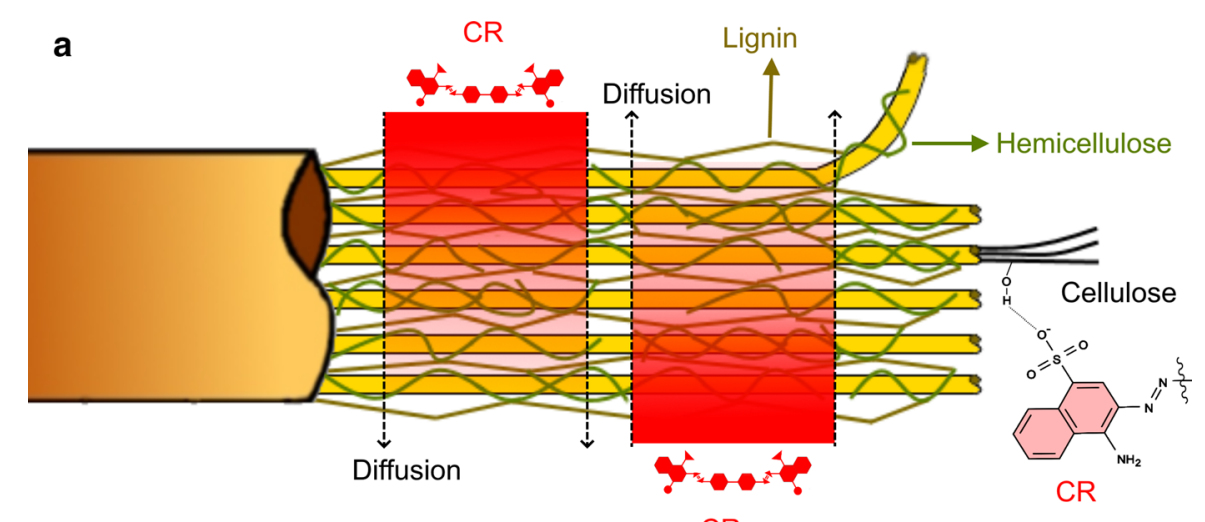

PDADMAC

$C R$

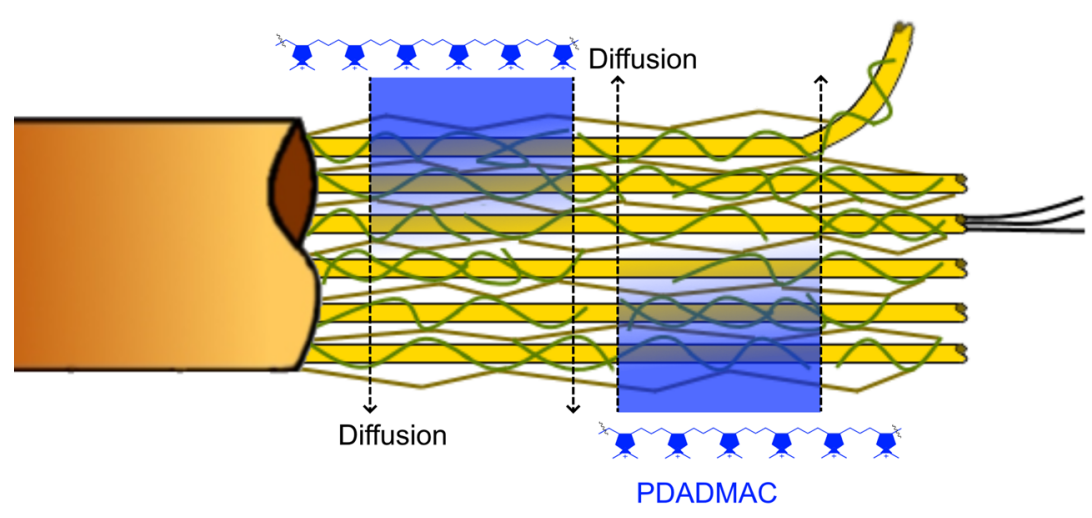

b

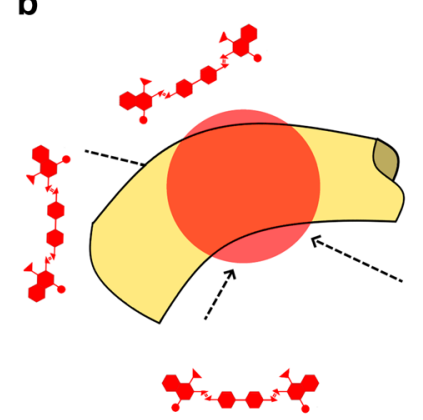

CR
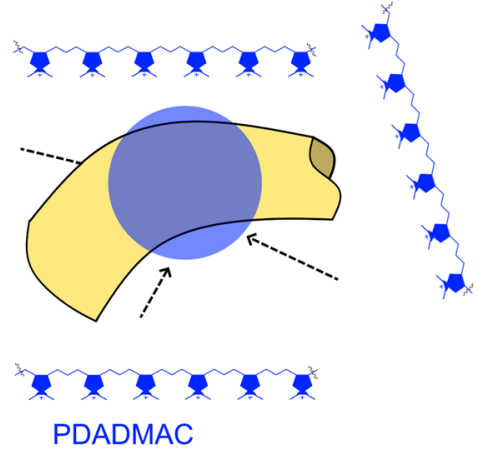

Fig. 5 Simplified schemes of the sorption and diffusion of CR and PDADMAC through lignocellulosic fibers (a) and fines (b)

usually mixed with cationic polyelectrolytes of high MW, most typically cationic polyacrylamides that resemble the structure of PDADMAC, in the wet-end of a paper machine (Petersen et al. 2013). A second motivation arises from the low residence times in the blend chest, in the machine chest and/or in other mixing operations that precede the formation of the paper web by filtration and thickening. Even low-MW solutes, such as alkenyl succinic anhydride, cannot diffuse through the center of a fiber because of kinetic limitations. Likewise, fiber-fiber, fine-fine and fiberfine interactions take place between outer surfaces, even if they are influenced by the degree of internal swelling.

Influence of $\mathrm{pH}$ and ionic strength

Whereas the interaction between PDADMAC and TMP has been studied in a deionized medium, this would not be the case of the wet end of an industrial 


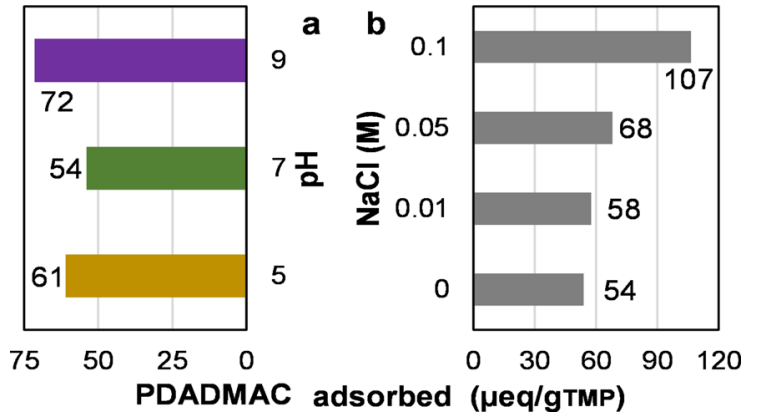

Fig. 6 Influence of $\mathrm{pH}$ (a) and salt concentration (b) on the adsorption of PDADMAC

paper machine. Different mills opt for different $\mathrm{pH}$ settings, often ranging from 5 (acid side) to 9 (alkaline side). Furthermore, ionic species are always present. Figure 6 presents the effect of $\mathrm{pH}$ and $\mathrm{NaCl}$ concentration on the amount of PDADMAC adsorbed per gram of pulp.

Both acid and alkaline conditions enhanced the adsorption of the polycation, likely by opening the structure of fibers to a certain extent through different mechanisms (swelling or cleavage at basic and acid $\mathrm{pH}$, respectively), thus easing diffusion through them. The enhancement was particularly high in the basic medium (Fig. 6a), given the increased polarization of $\mathrm{O}-\mathrm{H}$ bonds of hydroxyl groups, making them more prone to become attracted to PDADMAC units. Furthermore, while a $\mathrm{pH}$ of 9 is clearly below the $\mathrm{pK}_{\mathrm{a}}$ of sugar rings $(>11)$, it is high enough to deprotonate a notorious fraction, albeit less than 50\%, of the phenolic $-\mathrm{OH}$ groups of lignin (Ragnar et al. 2000).

Salt concentration affects adsorption in complex ways, taking into account that the screening effect reduces the intensity of Coulomb forces, and thus that of PDADMAC-TMP interactions. Nonetheless, the subsequent reduction in the charge density of PDADMAC provides some conformational flexibility and promotes rotations around $\mathrm{CH}_{2}-\mathrm{CH}_{2}$ bonds. First, the end-to-end length of PDADMAC chains becomes lower (Scheepers et al. 2021), easing their diffusion through fibers. Secondly, bent chains may present segments that are not in contact with any surface, allowing for a higher adsorption of PDADMAC than the one predicted from the monolayer hypothesis. These two effects result in increased PDADMAC adsorption (Fig. 6b). Still, Eq. 7 keeps being valid as long as the constant is recalculated for the specific set of conditions of the paper machine in each case.

\section{Conclusions}

Results supported the main hypothesis of the work, i.e., that a potentiometric titration to measure the cationic demand allows for a useful estimation of the SSA of lignocellulosic fibers. Such titration can be combined with a conductimetric one, aiming to discriminate between the amount of cationic polyelectrolyte ionically exchanged with carboxyl groups and the adsorption driven by ion-dipole interactions. In any case, this estimation of the SSA cannot replace the BET method $\left(\mathrm{N}_{2}\right)$ or dye sorption (in this work, Congo red), as it will yield lower values than these determinations, owing to the diffusion coefficient of PDADMAC being much lower. While this would imply an underestimation for other applications, in papermaking it is of great usefulness. Further, the proposed method was tested at increasing ionic strength and different $\mathrm{pH}$ values, revealing that both conditions had a notorious influence on the PDADMAC adsorption onto TMP fibers. In the case of ionic strength, it was attributed to the change on the persistence length of PDADMAC, which presumably modified the conformation of the polyelectrolyte during its adsorption. In terms of $\mathrm{pH}$, the differences were attributed to fiber swelling and cleavage, for basic and acid $\mathrm{pH}$. The SSA estimated from the methodology we present can be used as an indicator of refining, as shown by successfully correlating it to the Schopper-Riegler degree. Furthermore, it allows papermakers to differentiate between fibers, for which the values provided by our estimation are much lower those of dye sorption (e.g., 17.6 and $6.31 \mathrm{~m}^{2} \mathrm{~g}^{-1}$ in the case of 30,000 PFI revolutions), and fines, for which both methods attain similar results. More importantly, in light of the limitations to the diffusion of PDADMAC into fibers, the estimation of SSA from the cationic demand is thought to attain a valuable prediction of the behavior of the pulp with cationic polyacrylamides and in terms of inter-fiber interactions.

Acknowledgments Authors wish to acknowledge the financial support of the Spanish Ministry of Economy and Competitiveness to the Project NANOPROSOST, Reference 
CTQ2017-85654-C2-1-R. Marc Delgado-Aguilar is a Serra Húnter Fellow.

Authors' contributions All authors made substantial contributions to the conception of the work, the acquisition and interpretation of data, and writing. All authors approve the manuscript. All authors agree to be accountable for all aspects of the work in ensuring that questions related to the accuracy or integrity of any part of the work are appropriately investigated and resolved.

Funding Open Access funding provided thanks to the CRUECSIC agreement with Springer Nature. Financial support from the Spanish Ministry of Economy and Competitiveness is acknowledged.

Availability of data and materials The authors made all data public in the international repository Zenodo.

\section{Declarations}

Conflict of interest The authors declare that there is no conflict of interest and that they do not have competing interests.

Ethics approval Not applicable. No studies involving humans and/or animals.

Consent to participate Not applicable. No studies involving humans and/or animals.

Consent for publication Not applicable. No studies involving humans and/or animals.

Open Access This article is licensed under a Creative Commons Attribution 4.0 International License, which permits use, sharing, adaptation, distribution and reproduction in any medium or format, as long as you give appropriate credit to the original author(s) and the source, provide a link to the Creative Commons licence, and indicate if changes were made. The images or other third party material in this article are included in the article's Creative Commons licence, unless indicated otherwise in a credit line to the material. If material is not included in the article's Creative Commons licence and your intended use is not permitted by statutory regulation or exceeds the permitted use, you will need to obtain permission directly from the copyright holder. To view a copy of this licence, visit http://creativecommons.org/licenses/by/4.0/.

\section{References}

Aguado R, Moral A, López P et al (2016) Morphological analysis of pulps from orange tree trimmings and its relation to mechanical properties. Meas J Int Meas Confed. https://doi.org/10.1016/j.measurement.2016.06.063

Aguado R, Murtinho D, Valente AJM (2019) A broad overview on innovative functionalized paper solutions. Nord Pulp Pap Res J 34:395-416. https://doi.org/10.1515/npprj-20190036
Alves L, Medronho BF, Antunes FE et al (2015) On the role of hydrophobic interactions in cellulose dissolution and regeneration: Colloidal aggregates and molecular solutions. Colloids Surf A Physicochem Eng Asp 483:257-263. https://doi.org/10.1016/j.colsurfa.2015.03.011

Azevedo CA, Rebola SMC, Domingues EM et al (2020) Relationship between surface properties and fiber network parameters of eucalyptus kraft pulps and their absorption capacity. Surfaces. https://doi.org/10.3390/ surfaces 3030020

Barrera MC, Jorge M (2020) A Polarization-consistent model for alcohols to predict solvation free energies. J Chem Inf Model 60:1352-1367. https://doi.org/10.1021/acs.jcim. 9b01005

Carey FA, Sundberg RJ (2007) Advanced organic chemistry part a: structure and mechanisms, 2nd editio. Springer, New York

Carrasco F, Mutjé P, Pelach MA (1996) Refining of bleached cellulosic pulps: characterization by application of the colloidal titration technique. Wood Sci Technol 30:227-236

Darpentigny C, Molina-Boisseau S, Nonglaton G et al (2020) Ice-templated freeze-dried cryogels from tunicate cellulose nanocrystals with high specific surface area and anisotropic morphological and mechanical properties. Cellulose 27:233-247. https://doi.org/10.1007/s10570-019-02772-8

Delgado-Aguilar M, Tarrés Q, Pèlach MÀ et al (2015) Are cellulose nanofibers a solution for a more circular economy of paper products? Environ Sci Technol 49:12206-12213. https://doi.org/10.1021/acs.est.5b02676

El NA, Ragab S, El SA (2017) Rapid synthesis of cellulose triacetate from cotton cellulose and its effect on specific surface area and particle size distribution. Iran Polym J 26:261-272. https://doi.org/10.1007/s13726-017-0516-2

Espinosa E, Tarrés Q, Domínguez-Robles J et al (2018) Recycled fibers for fluting production: the role of lignocellulosic micro/nanofibers of banana leaves. J Clean Prod 172:233-238. https://doi.org/10.1016/j.jclepro.2017.10. 174

Filipova I, Serra F, Tarrés Q et al (2020) Oxidative treatments for cellulose nanofibers production: a comparative study between TEMPO-mediated and ammonium persulfate oxidation. Cellulose 27:10671-10688. https://doi.org/10. 1007/s10570-020-03089-7

Friedman HL, Mills R (1986) Hydrodynamic approximation for distinct diffusion coefficients. J Solution Chem 15:69-80. https://doi.org/10.1007/BF00646311

Gharehkhani S, Sadeghinezhad E, Kazi SN et al (2015) Basic effects of pulp refining on fiber properties - a review. Carbohydr Polym 115:785-803

González I, Boufi S, Pèlach MA et al (2012) Nanofibrillated cellulose as paper additive in eucalyptus pulps. BioResources 7:5167-5180

Hina K, Zou H, Qian W et al (2018) Preparation and performance comparison of cellulose-based activated carbon fibres. Cellulose 25:607-617. https://doi.org/10.1007/ s10570-017-1560-y

Inglesby MK, Zeronian SH (2002) Direct dyes as molecular sensors to characterize cellulose substrates. Cellulose 9:19-29. https://doi.org/10.1023/A:1015840111614 
Kaewprasit C, Hequet E, Abidi N, Gourlot JP (1998) Application of methylene blue adsorption to cotton fiber specific surface area measurement: part I. Methodology J Cotton Sci 2:164-173

Koponen A, Ekman A, Mattila K et al (2017) The effect of void structure on the permeability of fibrous networks. Transp Porous Media 117:247-259. https://doi.org/10.1007/ s11242-017-0831-2

Lecourt M, Sigoillot JC, Petit-Conil M (2010) Cellulase-assisted refining of chemical pulps: Impact of enzymatic charge and refining intensity on energy consumption and pulp quality. Process Biochem 45:1274-1278. https://doi.org/10.1016/j. procbio.2010.04.019

Mao R, Goutianos S, Tu W et al (2017) Comparison of fracture properties of cellulose nanopaper, printing paper and buckypaper. J Mater Sci 52:9508-9519. https://doi.org/10. 1007/s10853-017-1108-4

Moral A, Aguado R, Mutjé P, Tijero A (2016) Papermaking potential of Citrus sinensis trimmings using organosolv pulping, chlorine-free bleaching and refining. J Clean Prod. https://doi.org/10.1016/j.jclepro.2015.09.008

Moral A, Aguado R, Tijero A et al (2017) High-yield pulp from Brassica napus to manufacture packaging paper. BioResources. https://doi.org/10.15376/biores.12.2.2792-2804

Moser C, Backlund H, Lindström M, Henriksson G (2018) Xyloglucan for estimating the surface area of cellulose fibers. Nord Pulp Pap Res J 33:194-199. https://doi.org/10. 1515/npprj-2018-3035

Motamedian HR, Halilovic AE, Kulachenko A (2019) Mechanisms of strength and stiffness improvement of paper after PFI refining with a focus on the effect of fines. Cellulose 26:4099-4124. https://doi.org/10.1007/s10570-01902349-5

Petersen H, Radosta S, Vorwerg W, Kießler B (2013) Cationic starch adsorption onto cellulosic pulp in the presence of other cationic synthetic additives. Colloids Surf A Physicochem Eng Asp 433:1-8

Przybysz P, Dubowik M, Małachowska E et al (2020) The effect of the refining intensity on the progress of internal fibrillation and shortening of cellulose fibers. BioResources 15:1482-1499

Ragnar M, Lindgren CT, Nilvebrant N-O (2000) pKa-values of Guaiacyl and Syringyl phenols related to lignin. J Wood Chem Technol 20:277-305. https://doi.org/10.1080/ 02773810009349637

Ramarao BV (1999) Method and apparatus for determining pulp permeability and compressibility for controlling papermaking process

Robertson AA, Mason SG (1949) Specific surface of cellulose fibers by the liquid permeability method. Pulp Pap Mag Can 50:103-110
Rouger J, Mutjé P (1984) Correlation between the cellulose fibres beating and the fixation of a soluble cationic polymer. Br Polym J 16:83-86

Scheepers D, Chatillon B, Borneman Z, Nijmeijer K (2021) Influence of charge density and ionic strength on diallyldimethylammonium chloride (DADMAC)-based polyelectrolyte multilayer membrane formation. J Memb Sci 617:118619. https://doi.org/10.1016/j.memsci.2020. 118619

Serra-Parareda F, Tarrés Q, Pèlach MÀ et al (2021) Monitoring fibrillation in the mechanical production of lignocellulosic micro/nanofibers from bleached spruce thermomechanical pulp. Int J Biol Macromol 178:354-362

Strobl GR (2007) The physics of polymers, 3rd editio. Springer, Heidelberg

Tarrés Q, Oliver-Ortega H, Llop M et al (2016) Effective and simple methodology to produce nanocellulose-based aerogels for selective oil removal. Cellulose 23:3077-3088. https://doi.org/10.1007/s10570-016-10178

Tarrés Q, Oliver-Ortega H, Ferreira PJ et al (2018) Towards a new generation of functional fiber-based packaging: cellulose nanofibers for improved barrier, mechanical and surface properties. Cellulose 25:683-695. https://doi.org/ 10.1007/s10570-017-1572-7

Tarrés Q, Oliver-Ortega H, Boufi S et al (2020) Evaluation of the fibrillation method on lignocellulosic nanofibers production from eucalyptus sawdust: a comparative study between high-pressure homogenization and grinding. Int $\mathbf{J}$ Biol Macromol 145:1199-1207. https://doi.org/10.1016/j. ijbiomac.2019.10.046

Topgaard D, Söderman O (2002) Porous structure of cellulose fiber walls studied with NMR diffusometry. In: Fraissard J, Lapina $\mathrm{O}$ (eds) Magnetic resonance in colloid and interface science. Springer, Dordrecht, pp 631-635

Trizac E, Shen T (2016) Bending stiff charged polymers: the electrostatic persistence length. EPL 116:18007

Zhang H, Zhao C, Li Z, Li J (2016) The fiber charge measurement depending on the poly-DADMAC accessibility to cellulose fibers. Cellulose 23:163-173. https://doi.org/10. 1007/s10570-015-0793-x

Zhang J-Z, Peng X-Y, Liu S, et al (2019) The persistence length of semiflexible polymers in lattice monte carlo simulations. Polym 11

Zhao C, Zhang H, Zeng X et al (2016) Enhancing the inter-fiber bonding properties of cellulosic fibers by increasing different fiber charges. Cellulose 23:1617-1628. https://doi. org/10.1007/s10570-016-0941-y

Publisher's Note Springer Nature remains neutral with regard to jurisdictional claims in published maps and institutional affiliations. 